\title{
Wide-Field Telescope Alignment Using the Model-Based Method Combined with the Stochastic Parallel Gradient Descent Algorithm
}

\author{
Min $\mathrm{Li}^{1,2, *}$, Ang Zhang ${ }^{2}$, Junbo Zhang ${ }^{1,2}$ and Hao Xian ${ }^{1,2}$ \\ 1 Key Laboratory of Adaptive Optics, Chinese Academy of Sciences, Chengdu 610209, China; \\ zhangjunbo@ioe.ac.cn (J.Z.); xianhao@ioe.ac.cn (H.X.) \\ 2 Institute of Optics and Electronics, Chinese Academy of Sciences, Chengdu 610209, China; \\ zhangang@ioe.ac.cn \\ * Correspondence: limin414@mails.ucas.ac.cn
}

Citation: Li, M.; Zhang, A.; Zhang, J.; Xian, H. Wide-Field Telescope Alignment Using the Model-Based Method Combined with the Stochastic Parallel Gradient Descent Algorithm. Photonics 2021, 8, 463. https://doi.org/10.3390/photonics 8110463

Received: 13 September 2021

Accepted: 14 October 2021

Published: 22 October 2021

Publisher's Note: MDPI stays neutral with regard to jurisdictional claims in published maps and institutional affiliations.

Copyright: (c) 2021 by the authors. Licensee MDPI, Basel, Switzerland. This article is an open access article distributed under the terms and conditions of the Creative Commons Attribution (CC BY) license (https:// creativecommons.org/licenses/by/ $4.0 /)$.

\begin{abstract}
To acquire images with higher accuracy of wide-field telescopes, deformable mirrors with more than 100 actuators are used, making the telescope alignment more complex and timeconsuming. Furthermore, the position of the obscuration caused by the secondary mirror in the experiment system is changed with the difference of fields of view, making the response matrix of the deformable mirror different in various fields of view. To solve this problem, transfer functions corresponding to different fields of view are calculated according to the wavefront edge check and boundary conditions. In this paper, a model-based method combined with the stochastic parallel gradient descent (SPGD) algorithm is used. The experiment results show that our method can correct the aberrations with a high accuracy in both on-axis and off-axis fields, indicating that the effective actuators are well chosen corresponding to different fields of view.
\end{abstract}

Keywords: wide field of view; telescope alignment; deformable mirror; model-based method; SPGD algorithm

\section{Introduction}

Telescopes with wide fields of view are studied to explore a wider range of space. However, the final image quality is determined by telescope alignment accuracy. To correct the wavefront aberrations and acquire a high-resolution image, an adaptive element such as a deformable mirror (DM) [1] is usually employed. The higher the accuracy of the optical system that is required, the more actuators of deformable mirrors that are needed. Furthermore, the alignment of telescopes becomes more complex and time-consuming with the increase of actuators of deformable mirrors.

There are several methods used to correct the aberrations with the deformable mirror. All these methods can be classified into three categories: the direct wavefront sensor method [2,3], such as interferometers and Shack-Hartmann wavefront sensors; the wavefront sensor method based on images, such as the phase diversity method (PD) [4] and the phase retrieval method (PR) [5,6]; and the wavefront sensor-less method [7], which employs optimization algorithms such as genetic algorithm (GA) [8,9], the hill climbing method [10,11], the simulated annealing method (SA) [12], and the stochastic parallel gradient descent (SPGD) method $[13,14]$ to correct the aberrations according to merit functions. Compared with the direct wavefront sensor method and the wavefront sensor method based on images, the wavefront sensor-less method has the advantages of a concise system and simple principles as the correction processes do not require wavefront sensors to measure the aberrations and do not need wavefront reconstructions.

The wavefront sensor-less method can be divided into the model-free algorithm and the model-based algorithm $[15,16]$. The model-free algorithm is the most widely used 
method in previous works. However, the model-free algorithm may converge slowly, and sometimes it may get stuck in the local optimum, especially for deformable mirrors with more than 100 actuators. To solve this problem, the model-based algorithm was created. Different from the model-free algorithm, the model-based algorithm can converge much faster with only $\mathrm{N}+1$ iterations, where $\mathrm{N}$ is the number of corrected aberration terms [17]. Therefore, this kind of control algorithm has a great potential in real-time wavefront correction fields.

In this paper, a telescope with a wide field of view is aligned using a model-based method combined with the SPGD algorithm. A model-based method is used due to the large number of actuators, which may decrease the efficiency of the algorithm. In this paper, a deformable mirror with 357 actuators in the optical system is set as the entrance pupil with a tilted angle of $12.5^{\circ}$. As a result, the obscuration of the system caused by the secondary mirror in the position of the exit pupil changes with the different fields of view, making the response matrix of the deformable mirror different in various fields of view. The effective actuators corresponding to different fields of view are various, which means the transfer functions of the deformable mirror corresponding to different fields of view are changed. To solve this problem, transfer functions are calculated according to the fields of view. The process is introduced in detail in the Section 2.

The paper is organized as follows: in Section 2, the principle of the model-based method combined with the SPGD algorithm is introduced, and the transfer functions of the deformable mirror corresponding to different fields of view are analyzed; in Section 3, an experiment using the model-based SPGD algorithm with a Cassegrain telescope and a 357-element deformable mirror is described; and in Section 4, the conclusions are given.

\section{Theory}

\subsection{The Model-Based Method Combined with the SPGD Algorithm}

For wide field of view optical systems, the image quality in each field of view should satisfy the requirements of the applications. As a result, the higher the accuracy of the optical system that is required, the more actuators of the deformable mirror that are needed. The model-free methods based on the optimization of voltages of actuators become more complex and time-consuming. To improve the alignment efficiency, a model-based method combined with the SPGD algorithm is employed.

The SPGD algorithm is an iteration control method [18-20]. It applies small random perturbations to all control parameters simultaneously, and then evaluates the gradient variation of merit functions (J) [21]. The iterative formula of the SPGD algorithm is as follows [22].

$$
z^{k+1}=z^{k}-\gamma \times \delta J^{k} \times \delta z^{k}
$$

where, $\gamma$ is the gain coefficient, the sign of which is determined by merit functions. $\delta z$ is a small random perturbation, which has identical amplitudes and satisfies Bernoulli probability distribution. $\delta J$ is the variation of merit functions. $J$ is the merit function, such as strehl ratio, root mean square radius (RMS), encircled energy, and so on. $k$ is the number of iterations, and $z$ is the control variables.

For telescope optical systems with a point source at infinity, the input phase aberrations can be described by the function $\Phi(\rho, \theta)$. Here, $(\rho, \theta)$ are the polar coordinates in the pupil plane. The phase of the deformable mirror which is used to compensate for the aberrations of telescope optical systems can be expressed by the function $\Psi(\rho, \theta)$. The phase aberrations including the input phase aberrations $\Phi(\rho, \theta)$ and the phase aberrations of deformable mirrors $\Psi(\rho, \theta)$ can be described by a series of N Zernike polynomials [23], and each item can be expressed as $Z_{n}(\rho, \theta)$.

$$
\begin{aligned}
& \Phi(\rho, \theta)=\sum_{n=1}^{N} a_{n} z_{n}(\rho, \theta) \\
& \Psi(\rho, \theta)=\sum_{n=1}^{N} b_{n} z_{n}(\rho, \theta)
\end{aligned}
$$

where, $a_{n}$ and $b_{n}$ are the coefficients of the Zernike modes. 
The residual aberrations of the input wavefront whose aberrations are compensated by the deformable mirror can be represented by function $\mathrm{R}(\rho, \theta)$ and $\mathrm{R}(\rho, \theta)=\Phi(\rho, \theta)-\Psi(\rho, \theta)[24]$. According to the Fourier diffraction theory, the signal obtained by the detector can be expressed as follows [23].

$$
\mathrm{F}=I_{0}\left|\frac{1}{\pi} \int_{\theta=0}^{2 \pi} \int_{\rho=0}^{1} \exp \{j R(\rho, \theta)\} \rho d \rho d \theta\right|^{2}
$$

where, $I_{0}$ is the incident light power.

Here, we define $c_{n}$ as the coefficients of the residual wavefront error, and $c_{n}=a_{n}-b_{n}$. As a result, the far-field image can be expressed as follows [25].

$$
\mathrm{F}(\vec{c})=I_{0}\left|\frac{1}{\pi} \int_{\theta=0}^{2 \pi} \int_{\rho=0}^{1} \exp \left\{j \sum_{n=1}^{N} c_{n} Z_{n}(\rho, \theta)\right\} \rho d \rho d \theta\right|^{2}=I_{0} f(\vec{c})
$$

where, $\vec{c}=\left[\begin{array}{llllll}c_{1} & c_{2} & \ldots & \ldots & c_{n}\end{array}\right]^{T} \cdot f(\vec{c})$ can be approximately expressed as follows due to the orthogonality of the Zernike modes [25].

$$
f(\vec{c}) \approx 1-|\vec{c}|^{2}
$$

From the above equation, we know that the global maximum can be obtained when the coefficient $\vec{c}$ is 0 .

As a result, the SPGD algorithm aims at optimizing the parameter $\vec{c}$ to attain a minimum. Experiments are carried out according to the principle of the alignment method.

\subsection{Transfer Functions of the Deformable Mirror Corresponding to Different Fields of View}

For some telescopes, the large fields of view are obtained by scanning. For example, to acquire a $3^{\circ}$ field of view, a telescope with a $1^{\circ}$ field of view should scan at least three times. To solve this problem, a telescope with a wide field of view $3.2^{\circ}$ is designed, which can look at the $3^{\circ}$ field of view without scanning. However, the image quality in off-axis fields is poor, which cannot satisfy the requirement of resolutions. Therefore, a deformable mirror is employed to correct the aberrations.

In this paper, experiments are carried out using a wide-field optical system, whose entrance pupil is set at the deformable mirror with a tilted angle of $12.5^{\circ}$. Thus, the obscuration caused by the secondary mirror shifts with changes of fields of view, leading to the various effective actuators corresponding to different fields of view. This is because the voltages of actuators obscured by the secondary mirror are usually very large, which have no sense for aberration correction and may introduce high order aberrations. Therefore, the effective actuators must be calculated corresponding to the different fields of view. That means the transfer functions of the deformable mirror for different fields of view are not the same.

The telescope is a wide-field system with a field of view of $\Phi 3.2^{\circ}$. The positions of obscuration are different with the changes of fields of view.

In Figure 1, wavefront maps corresponding to different fields of view between $-1.6^{\circ}$ and $1.6^{\circ}$ are shown. The wavefront maps show that the image quality in the on-axis field of view is good with $0.0029 \lambda(\lambda=0.55 \mu \mathrm{m})$ wavefront RMS. However, image qualities in other fields of view become worse, especially the images in the edge fields of view. In this paper, the aberrations are corrected using the deformable mirror. The figure indicates that the obscuration centers of the wavefront maps change with the difference of fields of view. To measure the offsets of obscuration accurately, edge check and circle fitting are implemented. The results are shown in Table 1. 


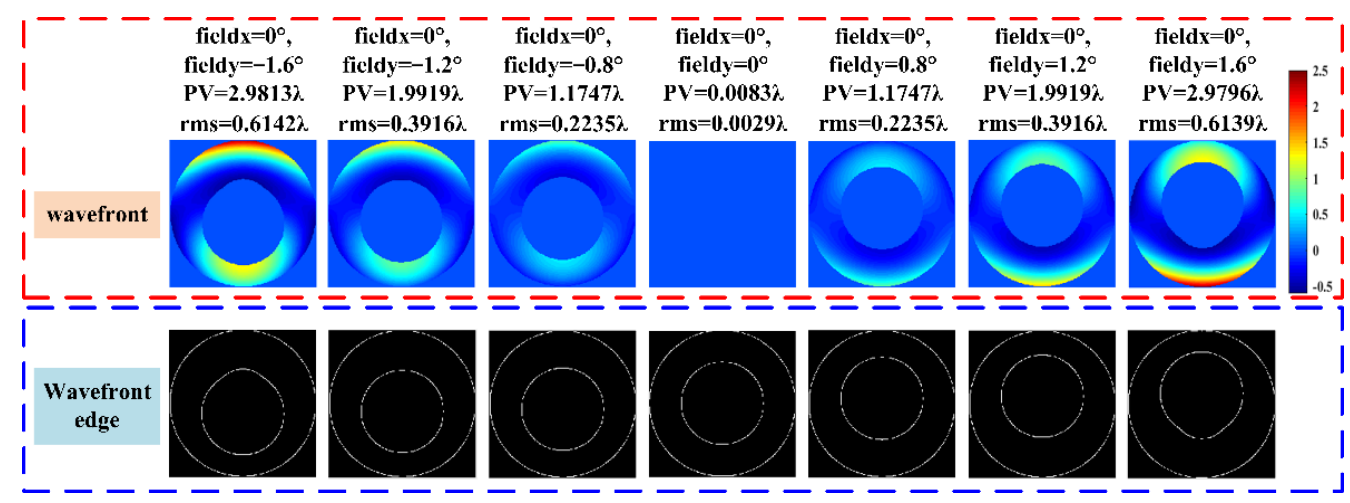

Figure 1. Wavefront corresponding to different fields of view.

Table 1. Results of the circle fitting corresponding to wavefront in different fields of view.

\begin{tabular}{cccccc}
\hline Field & $\mathbf{c x}_{\text {out }} / \mathbf{m m}$ & $\mathbf{c y}_{\text {out }} / \mathbf{m m}$ & $\mathbf{c x}_{\text {in }} / \mathbf{m m}$ & $\mathbf{c y}_{\mathbf{i n}} / \mathbf{m m}$ & Standard Deviation $/ \mathbf{m m}$ \\
\hline$\left(0^{\circ},-1.6^{\circ}\right)$ & 18.58 & 18.47 & 18.58 & 20.88 & 2.41 \\
$\left(0^{\circ},-1.2^{\circ}\right)$ & 18.58 & 18.47 & 18.58 & 20.42 & 1.95 \\
$\left(0^{\circ},-0.8^{\circ}\right)$ & 18.58 & 18.47 & 18.58 & 19.77 & 1.30 \\
$\left(0^{\circ}, 0^{\circ}\right)$ & 18.58 & 18.47 & 18.58 & 18.45 & 0.02 \\
$\left(0^{\circ}, 0.8^{\circ}\right)$ & 18.58 & 18.47 & 18.58 & 17.13 & 1.34 \\
$\left(0^{\circ}, 1.2^{\circ}\right)$ & 18.58 & 18.47 & 18.58 & 16.48 & 1.99 \\
$\left(0^{\circ}, 1.6^{\circ}\right)$ & 18.58 & 18.47 & 18.58 & 16.09 & 2.38 \\
\hline
\end{tabular}

In Table $1,\left(\mathrm{cx}_{\text {out }}, \mathrm{cy}_{\text {out }}\right)$ is the center of the exit pupil, while $\left(\mathrm{cx}_{\mathrm{in}}, \mathrm{cy}\right.$ in $)$ is the center of the obscuration caused by the secondary mirror. Positions of the obscuration are different with the changes of fields of view. The effective actuators corresponding to different fields of view are not the same. As a result, there are various transfer functions related to the different fields of view. Thus, the aberrations may not be corrected precisely when aberrations are corrected in the off-axis fields of view using the transfer function corresponding to the on-axis field of view. To analyze the effect of transfer function changes, simulations are implemented. Three kinds of misalignments with different aberrations are given based on the Zernike polynomial, and the different Zernike coefficients are shown in Table 2.

Table 2. Zernike coefficients corresponding to three misalignments.

\begin{tabular}{ccccccc}
\hline Zernike Order & $\mathbf{Z 4 / \lambda}$ & $\mathbf{Z 5 / \lambda}$ & $\mathbf{Z 6 / \lambda}$ & $\mathbf{Z 7 / \lambda}$ & $\mathbf{Z 8 / \lambda}$ & $\mathbf{Z 1 1 / \lambda}$ \\
\hline Misalignment 1 & 1 & 1 & -1 & -1 & 1 & 1 \\
Misalignment 2 & 0.3 & -0.2 & 0.8 & 0.1 & -0.6 & -0.5 \\
Misalignment 3 & -0.1 & 0.4 & -0.5 & 0.2 & 0.3 & -0.2 \\
\hline
\end{tabular}

Here, the defocus aberration (Z4), the astigmatism aberrations (Z5) and (Z6), the coma aberrations (Z7) and (Z8), and the spherical aberration (Z11) are given. Three misalignments are simulated.

The aberrations cannot be corrected precisely if the transfer functions of the deformable mirror do not match with the fields of view. The root mean square (rms) of the residual wavefront error is calculated after aberrations are corrected using transfer functions corresponding to different fields of view. The corrected results are compared in Figure 2. 


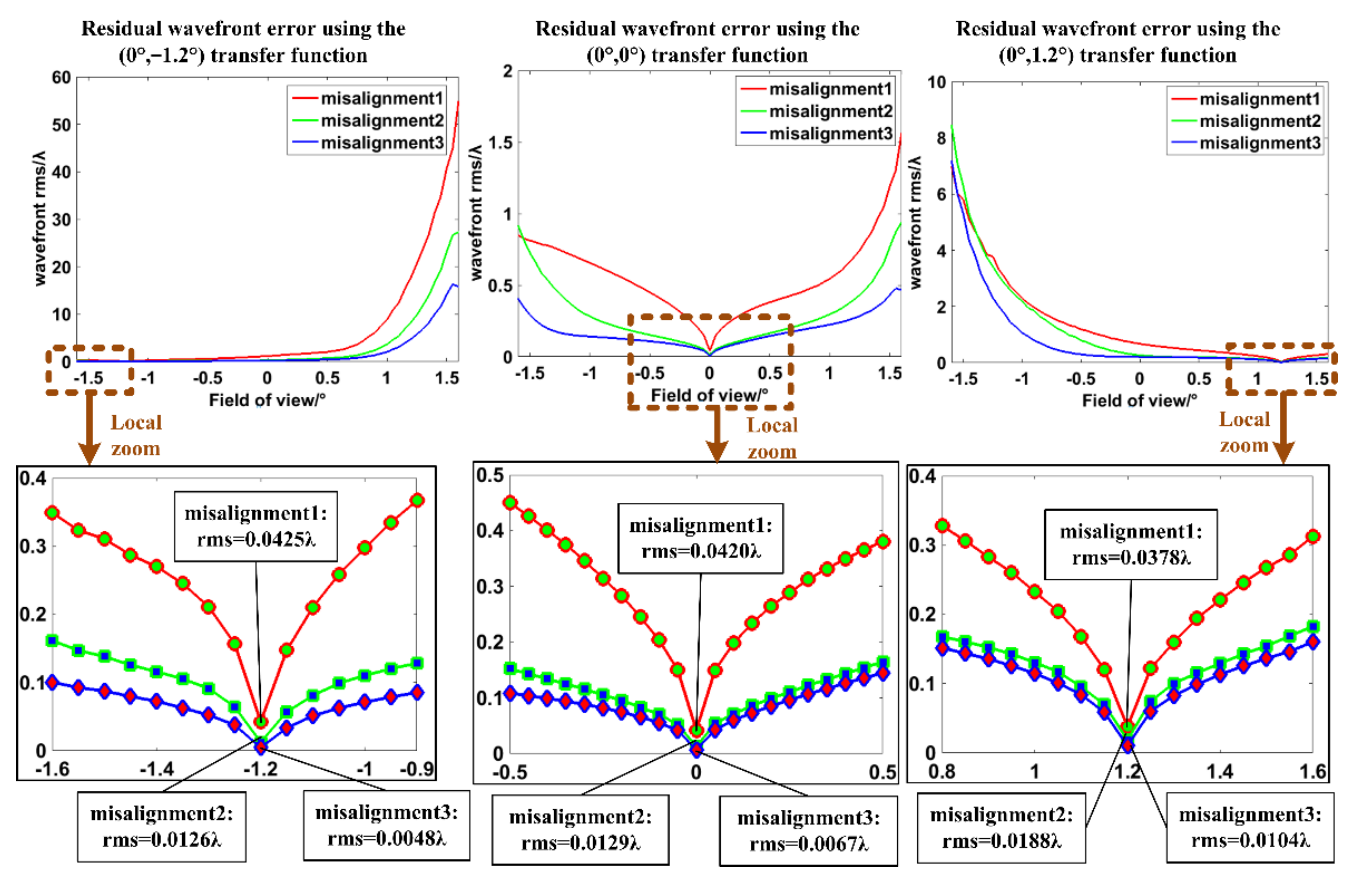

Figure 2. The residual wavefront errors after aberration correction using different transfer functions.

Figure 2 shows the residual wavefront aberrations corresponding to misalignments 1 , 2 , and 3 in different fields of view when the aberrations are corrected using the transfer functions of the deformable mirror in fields of view $\left(0^{\circ},-1.2^{\circ}\right),\left(0^{\circ}, 0^{\circ}\right)$, and $\left(0^{\circ}, 1.2^{\circ}\right)$. The image in the first column in Figure 2 shows the residual wavefront aberrations using the transfer function in the field of view $\left(0^{\circ},-1.2^{\circ}\right)$. The results show that the aberrations in field of view $\left(0^{\circ},-1.2^{\circ}\right)$ are well corrected, with residual wavefront errors as $0.0425 \lambda$ for misalignment $1,0.0126 \lambda$ for misalignment 2 , and $0.0048 \lambda$ for misalignment 3 . However, aberrations in other fields of view are not well compensated. The second column and the third column show the residual aberrations corresponding to different fields of view when the aberrations are corrected using the transfer functions of the deformable mirror in fields of view $\left(0^{\circ}, 0^{\circ}\right)$ and $\left(0^{\circ}, 1.2^{\circ}\right)$. The results show that aberrations can be well corrected when the transfer function corresponding to the field of view is used, and the aberrations remain large when the transfer functions do not match with the fields of view. This is because the effective actuators of the deformable mirror are not selected correctly. The large residual wavefront error is caused by the actuators which should not work or need to work but did not in the effective region of the field of view.

To solve this problem, the edge check results of the wavefront at the position of the exit pupil are used to calculate the transfer functions of the deformable mirror to match with different fields of view. The aberrations are also corrected using the effective transfer functions chosen by the corresponding fields of view. The results are shown in Figure 3.

Figure 3 shows the aberration corrected results using the transfer functions corresponding to each field of view in the condition of three kinds of misalignments. That means, for aberrations correction in the field $\left(0^{\circ},-1.6^{\circ}\right)$, the transfer function of the deformable mirror is calculated according to the exit pupil in the field $\left(0^{\circ},-1.6^{\circ}\right)$, and for aberrations correction in the field $\left(0^{\circ}, 1.6^{\circ}\right)$, the transfer function of the deformable mirror is calculated according to the exit pupil in the field $\left(0^{\circ}, 1.6^{\circ}\right)$. In this case, the residual wavefront errors corresponding to different fields of view for three kinds of misalignments are less than $0.05 \lambda$, indicating that the aberrations are well compensated when the right transfer functions matching with the fields of view are used. As a result, the transfer functions of the deformable mirror should be employed according to different fields of view. 


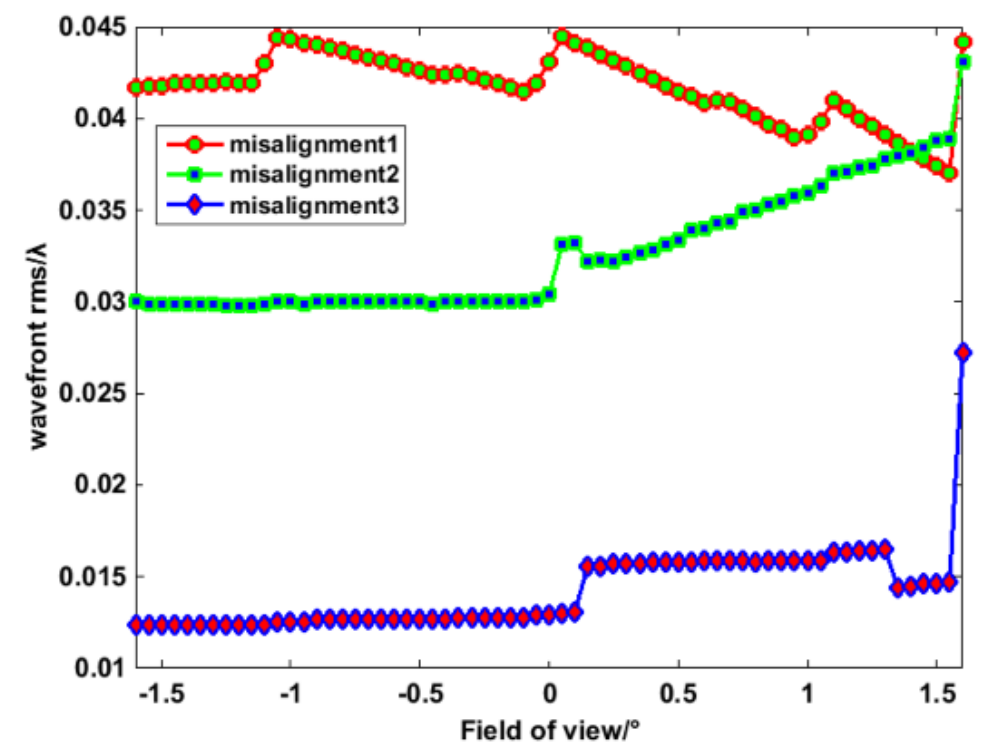

Figure 3. Residual wavefront errors in different fields of view for three misalignments.

The second mirror is supported by trusses which are evenly distributed. As a result, the exit pupil in conjugate position contains the shadow of trusses. The voltages calculated based on the transfer functions of the deformable mirror may exist large numbers in the position of the edge and trusses. To solve this problem, a threshold is set to filtrate the voltages.

As shown in Figure 4, the transfer functions of the deformable mirror are calculated with the following processes. Firstly, the transfer function in the on-axis field of view which can also be the called response matrix of the deformable mirror should be measured using the auto-collimation method or wavefront sensor method. If the wavefront sensor method is used, a parallel light source and a wavefront sensor with a collimating system as well as a down-collimator should be used. Moreover, the collimating system should be aligned first. However, the auto-collimation method only requires an interferometer and a flat mirror. As a result, the transfer function is measured by the auto-collimation method with a $4 \mathrm{D}$ interferometer in this paper. Secondly, the fields of view are determined according to the centroid positions of the images obtained on the detector. Because the telescope is a system with a wide field of view, images in both on-axis and off-axis fields of view should be corrected. As the figure shows, the image quality in different fields of view is corrected. Thirdly, the effective actuators corresponding to different fields of view are chosen using the wavefront edge check. The effective actuators are different for the reason that positions of the obscuration caused by the secondary mirror become various with the changes of fields of view. The actuators in the shadow range should not be used when the aberrations are corrected. Then, the transfer functions corresponding to different fields of view should be generated according to the effective actuators. Finally, the telescope is aligned using the model-based method combined with the SPGD algorithm. 


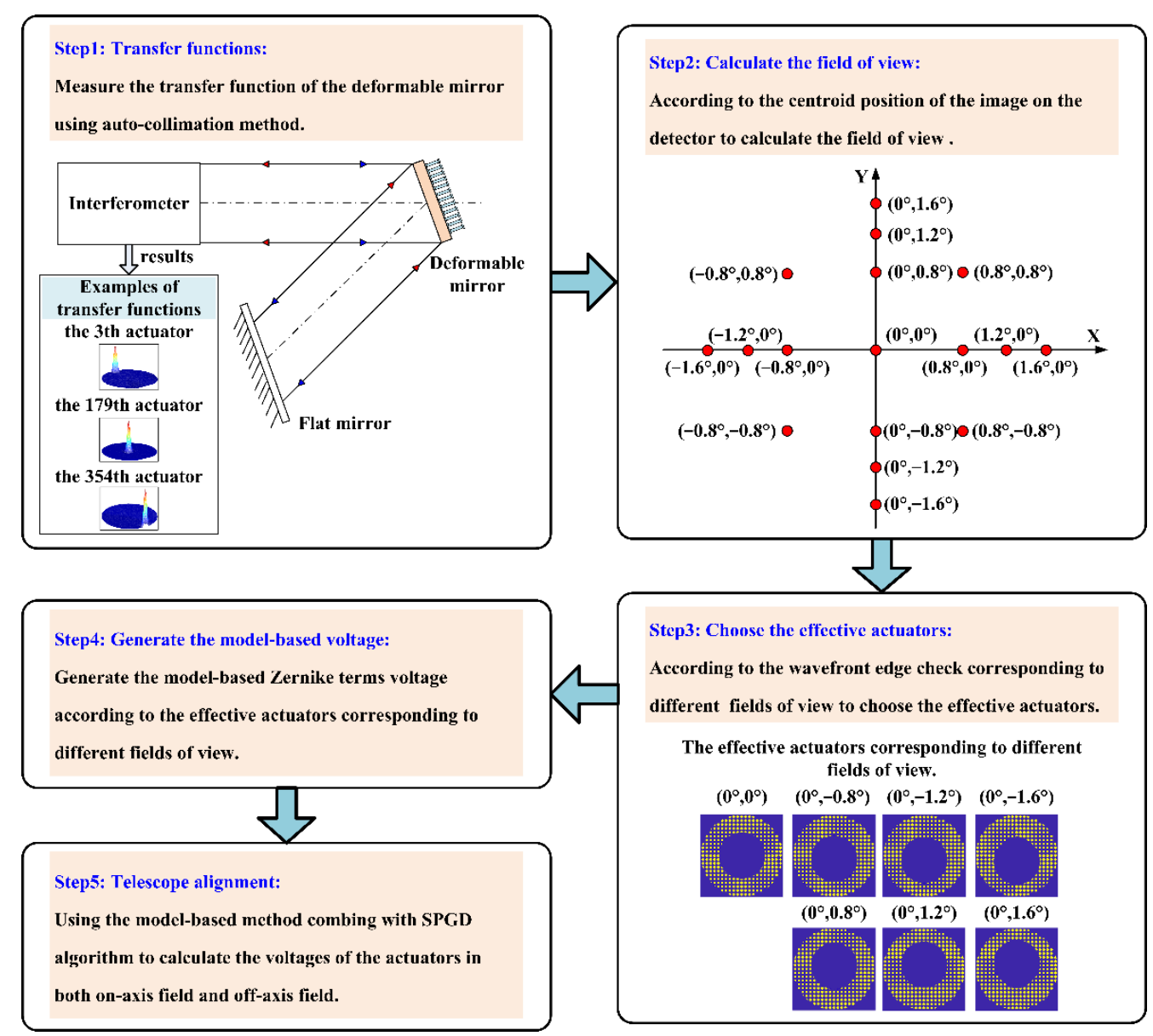

Figure 4. Calculation principles of transfer functions corresponding to different fields of view.

\section{Experiments}

In this paper, a telescope with a wide field of view $3.2^{\circ}$ is employed. The F\# is 13.6, and the effective optical aperture is $60 \mathrm{~mm}$ to avoid the effect of atmosphere turbulence. To correct the aberrations in different fields of view, a deformable mirror with 357 actuators is employed. The aberrations caused by the deformable mirror are calibrated, and flat voltages which can compensate for the aberrations of deformable mirror are calculated. To acquire high resolution images in both the on-axis field and the off-axis fields, several fields of view are corrected. In this paper, four correction examples are given.

\subsection{Experiment System}

For the model-based optimization method, the control variables are coefficients of the Zernike modes. The experiment system and correction processes are shown in Figure 5.

The experiment system includes a deformable mirror, a primary mirror, and a secondary mirror. The deformable mirror is the entrance pupil, which can change the surface morphology using the actuators controlled by the DM controller. The model-based method combined with the SPGD algorithm is introduced in this figure. The image of the optical system is acquired by the detector, of which the merit function $(J)$ is calculated. If the merit function satisfies the image quality requirement, the telescope alignment is finished. Otherwise, the model-based method will be carried out to correct the aberrations. The variables of the alignment process are Zernike coefficients $\left(Z=\left[z_{1}, z_{2}, \ldots \ldots, z_{N}\right]\right)$ and $N$ stands for the items of the Zernike modes. In this paper, 37 Zernike items are calculated, which means $\mathrm{N}$ is equal to $37 . \mathrm{M}$ is the response matrix of the deformable mirror, which can be calculated according to the method described in Section 2.2. $U$ is the voltages of the deformable mirror actuators, which can be expressed as $U=\left[u_{1}, u_{2}, \ldots \ldots, u_{m}\right]$. Here, $m$ is 
the number of actuators. In this paper, a deformable mirror with 357 actuators is used, so $\mathrm{m}$ is equal to 357. Then, each Zernike coefficient is disturbed by a small stochastic disturbance $\delta z$, and the merit function is calculated after each disturbance until all the Zernike modes are optimized. To improve the accuracy of the estimations, binary-side perturbation is used. The new Zernike coefficients are calculated by the iterative formula $z_{n}=z_{n}-\gamma \delta J \delta z$. After all the Zernike coefficients are optimized, the new voltages of the deformable mirror corresponding to different actuators are calculated by equation $U=M^{*} Z$. The surface morphology of the deformable mirror changes when the voltages $U$ are updated. These processes should be repeated until the image quality obtained by the detector satisfies the criterion.

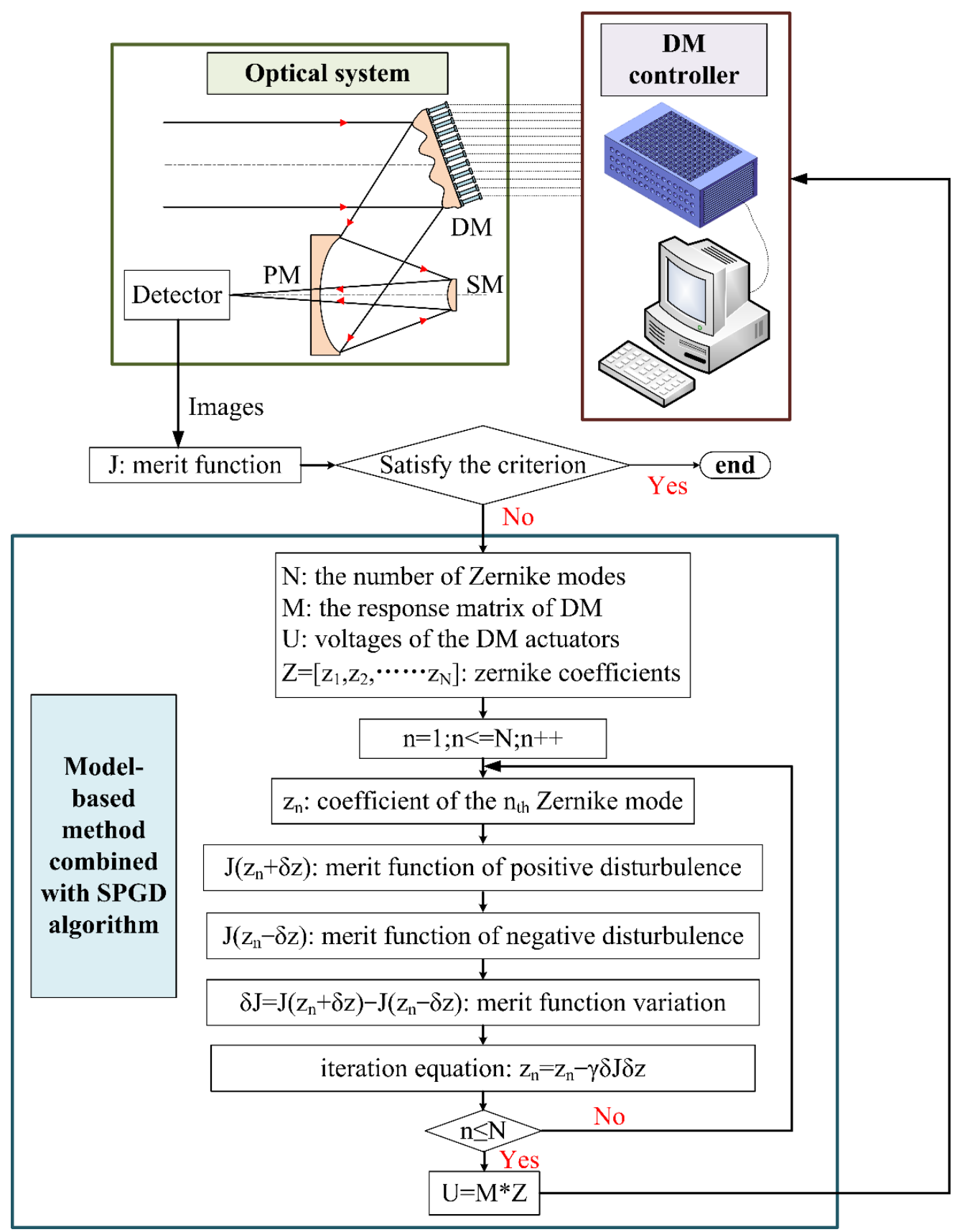

Figure 5. The experiment system and correction processes.

To match the resolution of the optical system, a splicing screen with sixteen computer monitors arranged in a $4 \times 4$ pattern is employed. The resolution of each computer monitor 
is 3840 pixels $\times 2160$ pixels, and the size of each pixel is $0.15 \mathrm{~mm}$, satisfying the resolution demands of the optical system and the detection system. The point sources are simulated using monitors with $4 \mathrm{~K}$ resolution positioned $25 \mathrm{~m}$ away from the optical system. Because the brightness of the monitors is not enough, the images obtained by the detector are badly affected by noise. For this reason, strehl ratio is used as the merit function. In the experiments, four targets with aberrations are corrected. The target distribution is shown in Figure 6.

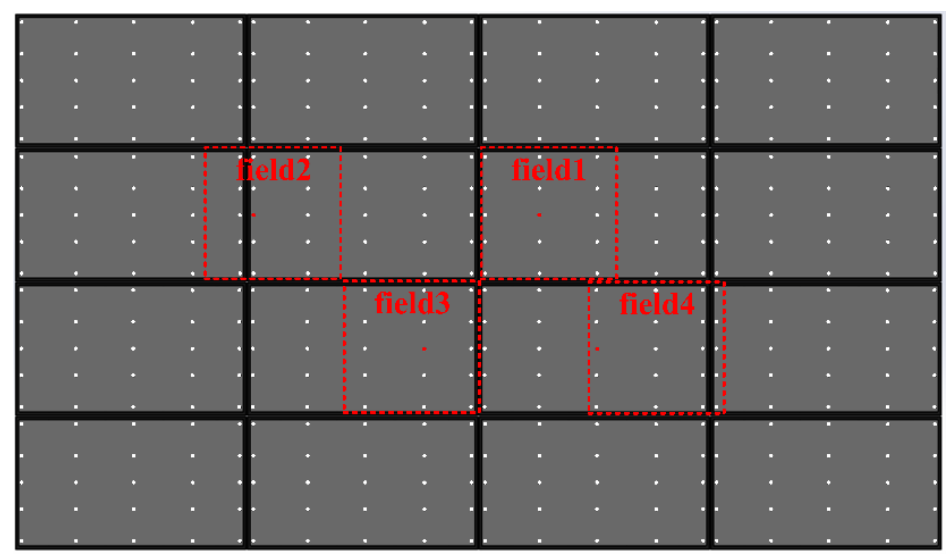

Figure 6. Targets distribution.

As shown in the figure, the red rectangles are four effective field areas. The red points are the targets, according to which the aberrations should be compensated. The other white points are the images in the adjacent fields of view. The point targets are displayed with computer monitors whose intensities are not very strong and the distance between the objects and the optical system is $25 \mathrm{~m}$, which is too far to be detected. Also, the stray light from the environment is strong. As a result, the images acquired by the detector have a high level of noise. To correct the aberrations of the optical system using the model-based SPGD algorithm, firstly, the noise images should be preprocessed. Secondly, to avoid the effect of noise on the convergence speed and stability, the strehl ratio should be used as the merit function.

\subsection{Experiment Results}

In this paper, the normalized strehl ratio is employed as the merit function to decrease the effect of the noise. For different fields of view, the convergence curves are shown in Figure 7.

As can be seen from Figure 7, the alignment processes for different fields of view converge after 30 to 50 iterations and the normalized strehl ratio settles above 0.9 . The speed of convergence depends on the parameters of the SPGD algorithm, such as disturbance, gain, merit functions, and so on.

The radius and the root mean square radius (RMS) of the far field images are also calculated to analyze the image quality. The RMS is defined by the following equation [26].

$$
\begin{gathered}
\mathrm{RMS}=\frac{\iint \sqrt{\left(x-x_{0}\right)^{2}+\left(y-y_{0}\right)^{2}} I(x, y) d x d y}{\iint I(x, y) d x d y} \\
x_{0}=\frac{\iint x \cdot I(x, y) d x d y}{\iint I(x, y) d x d y}, \quad y_{0}=\frac{\iint y \cdot I(x, y) d x d y}{\iint I(x, y) d x d y}
\end{gathered}
$$

where, $(x, y)$ are the coordinates of the images. $I(x, y)$ is the intensity, and $\left(x_{0}, y_{0}\right)$ are the centroids of images. 

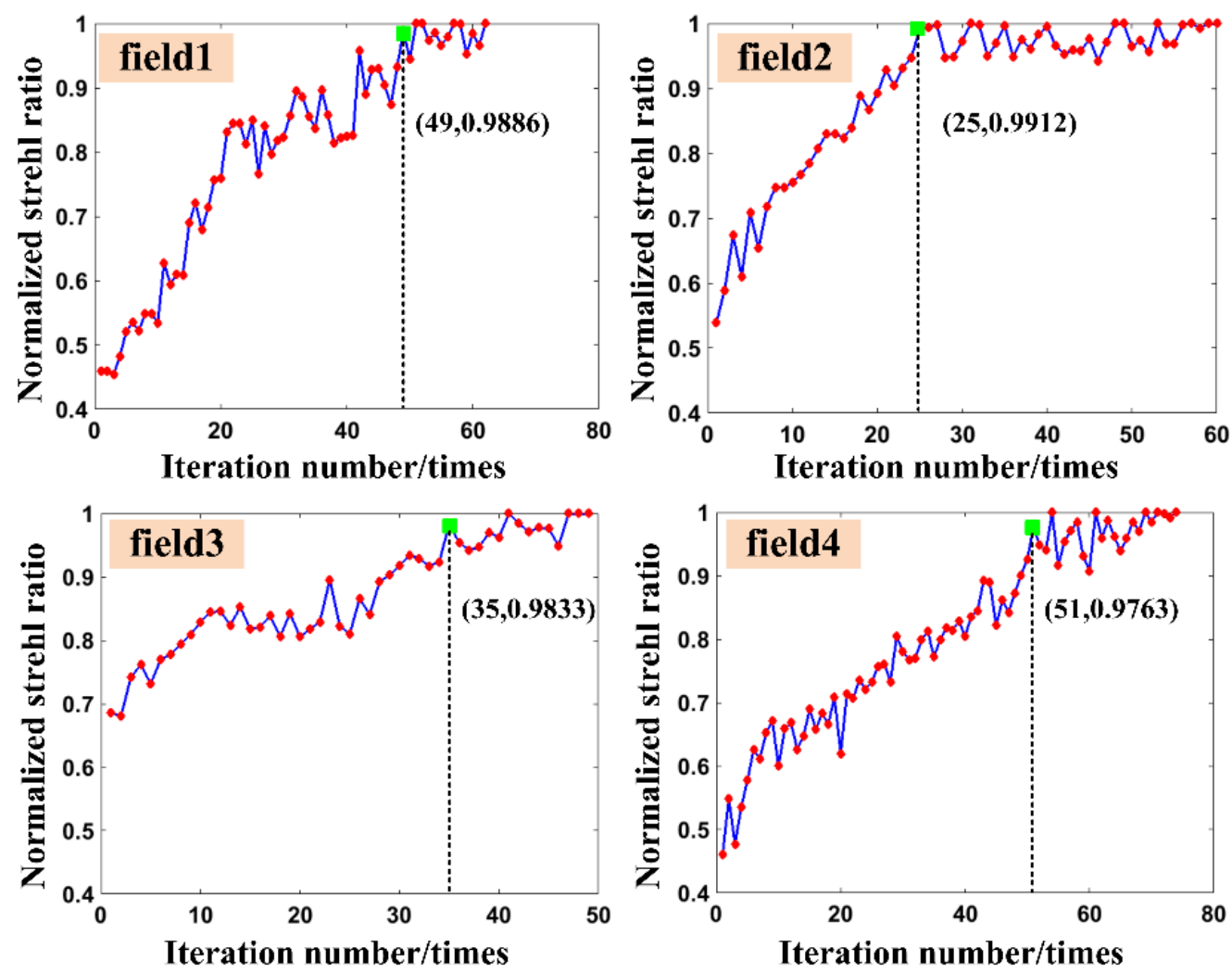

Figure 7. Convergence curves corresponding to different fields of view.

The misaligned images and corrected images are shown in Figure 8.

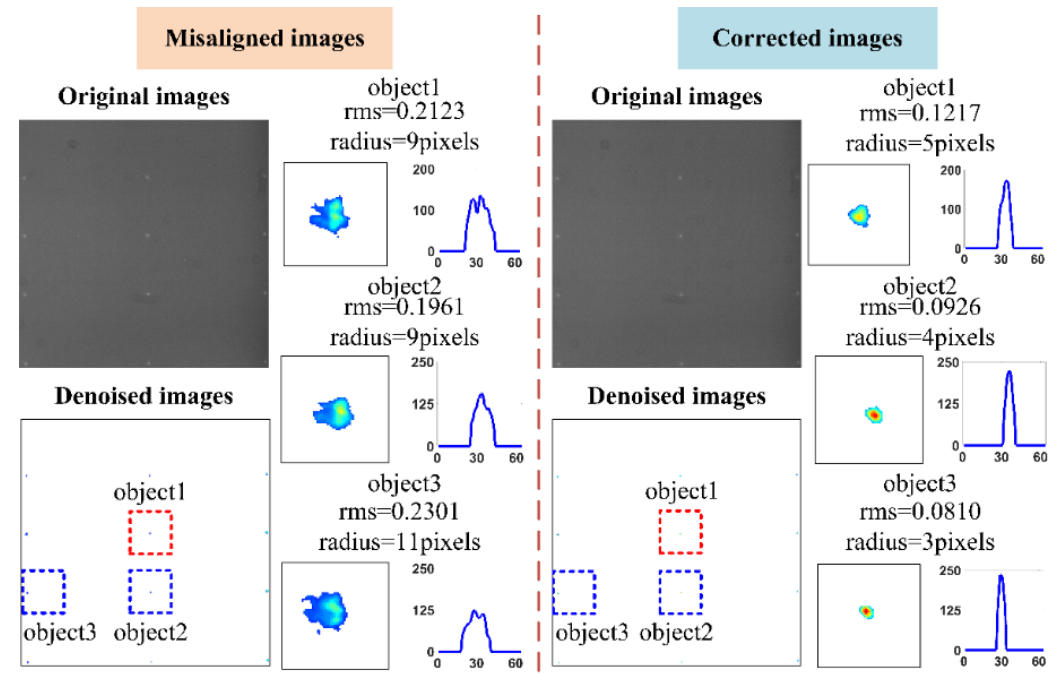

(a) field 1

Figure 8. Cont. 


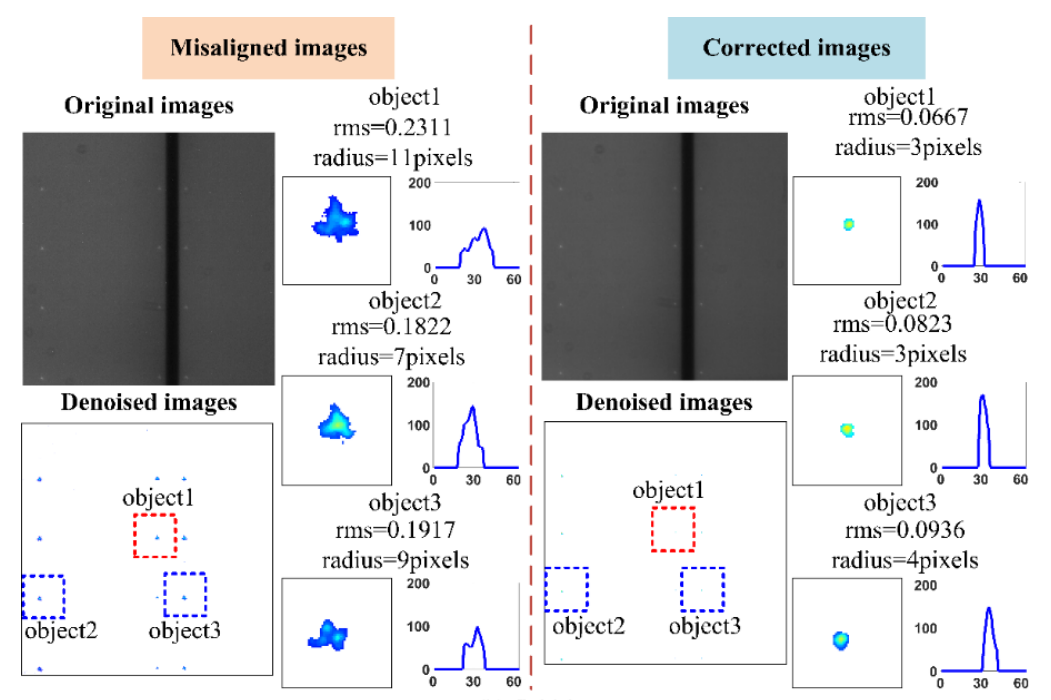

(b) field 2

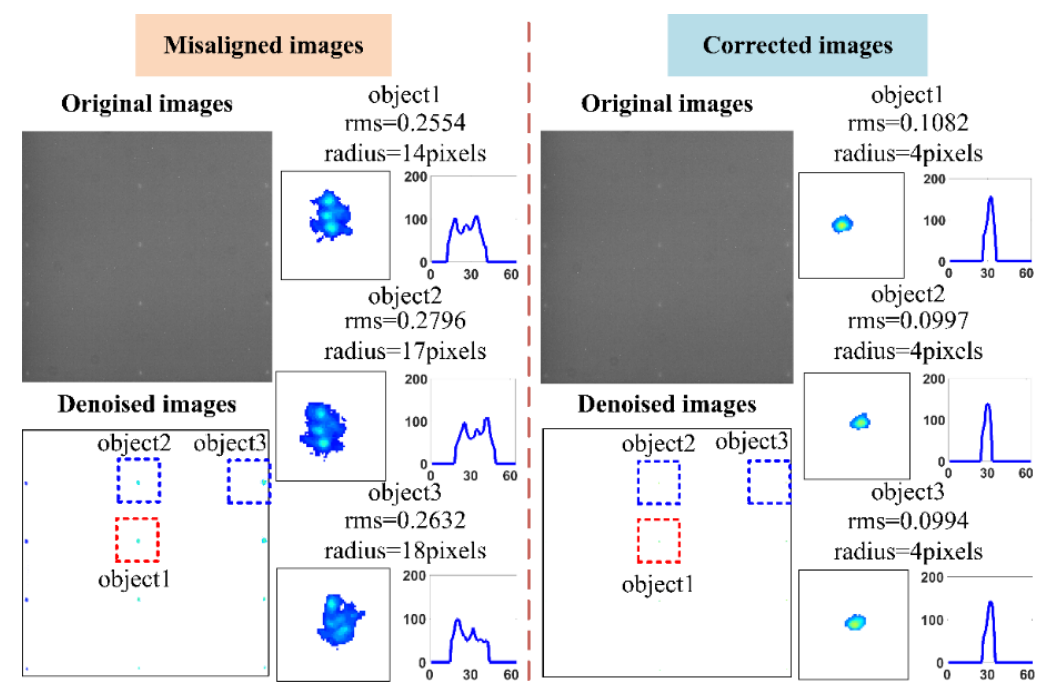

(c) field 3

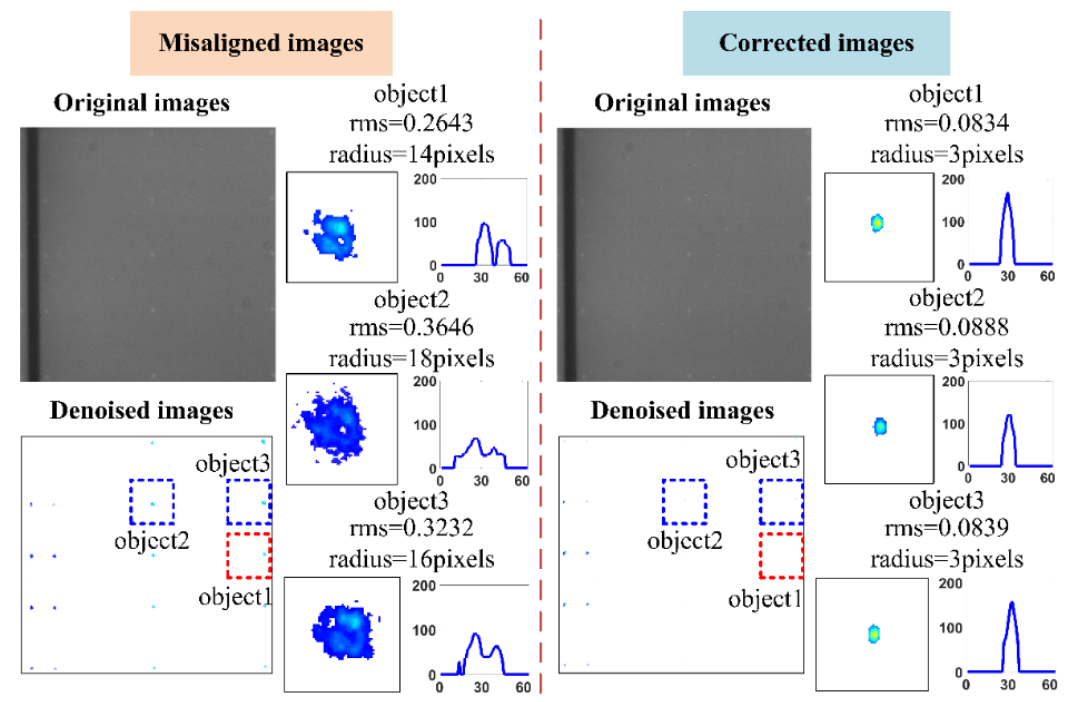

(d) field 4

Figure 8. Misaligned and corrected images of (a) field 1, (b) field 2, (c) field 3, (d) and field 4. 
Three targets in each field of view are calculated. The targets in red boxes are the objects according to which aberrations are corrected, while the targets in blue boxes are the objects in the adjacent fields of view. The root mean square radius (RMS) and the radius of the misaligned images and corrected images are shown in Table 3.

Table 3. Root mean square radius (RMS) and radius of misaligned and corrected images.

\begin{tabular}{|c|c|c|c|c|c|c|}
\hline \multicolumn{7}{|c|}{ RMS } \\
\hline \multirow[b]{2}{*}{ Field } & \multicolumn{2}{|c|}{ Object 1} & \multicolumn{2}{|c|}{ Object 2} & \multicolumn{2}{|c|}{ Object 3} \\
\hline & $\begin{array}{l}\text { Misaligned } \\
\text { Images }\end{array}$ & $\begin{array}{l}\text { Corrected } \\
\text { Images }\end{array}$ & $\begin{array}{l}\text { Misaligned } \\
\text { Images }\end{array}$ & $\begin{array}{l}\text { Corrected } \\
\text { Images }\end{array}$ & $\begin{array}{l}\text { Misaligned } \\
\text { Images }\end{array}$ & $\begin{array}{l}\text { Corrected } \\
\text { Images }\end{array}$ \\
\hline 1 & 0.2123 & 0.1217 & 0.1961 & 0.0926 & 0.2301 & 0.0810 \\
\hline 2 & 0.2311 & 0.0667 & 0.1822 & 0.0823 & 0.1917 & 0.0936 \\
\hline 3 & 0.2554 & 0.1082 & 0.2796 & 0.0997 & 0.2632 & 0.0994 \\
\hline 4 & 0.2643 & 0.0834 & 0.3646 & 0.0888 & 0.3232 & 0.0839 \\
\hline \multicolumn{7}{|c|}{ Radius/pixels } \\
\hline \multirow[b]{2}{*}{ Field } & \multicolumn{2}{|c|}{ Object 1} & \multicolumn{2}{|c|}{ Object 2} & \multicolumn{2}{|c|}{ Object 3} \\
\hline & $\begin{array}{l}\text { Misaligned } \\
\text { Images }\end{array}$ & $\begin{array}{l}\text { Corrected } \\
\text { Images }\end{array}$ & $\begin{array}{l}\text { Misaligned } \\
\text { Images }\end{array}$ & $\begin{array}{l}\text { Corrected } \\
\text { Images }\end{array}$ & $\begin{array}{l}\text { Misaligned } \\
\text { Images }\end{array}$ & $\begin{array}{l}\text { Corrected } \\
\text { Images }\end{array}$ \\
\hline 1 & 9 & 5 & 9 & 4 & 11 & 3 \\
\hline 2 & 11 & 3 & 7 & 3 & 9 & 4 \\
\hline 3 & 14 & 4 & 17 & 4 & 18 & 4 \\
\hline 4 & 14 & 3 & 18 & 3 & 16 & 3 \\
\hline
\end{tabular}

As can be seen from the results, the aberrations are well corrected using the modelbased SPGD algorithm. Both the RMS and radius of spots are decreased. The image quality after correction satisfies the requirement with residual wavefront errors less than $0.1 \lambda$. Furthermore, not only are the aberrations in the corrected field of view well compensated, but also the aberrations in the neighbor fields of view are corrected. This is because these fields of view are in the isoplanatic region.

\section{Conclusions}

For wide field of view telescopes, the aberrations in both on-axis field and off-axis fields should be corrected. This paper focuses on two problems of the telescope alignment. One problem is the position of the obscuration caused by the secondary mirror changes with different fields of view, making the transfer functions of the deformable mirror change with the fields of view. The other problem is that the deformable mirror has more than 100 actuators, making the alignment process more complex and time-consuming.

To solve the first problem, the transfer functions corresponding to different fields of view are calculated according to the wavefront edge check at the position of the exit pupil. To solve the second problem, a model-based method combined with the SPGD algorithm is used to correct the aberrations to improve the convergence efficiency. The experiment results show that the aberrations are well compensated whether the images are in the on-axis field or in the off-axis fields, verifying that the effective actuators are well chosen corresponding to different fields of view and the showing the feasibility of our method.

Author Contributions: Conceptualization, M.L., A.Z., J.Z., and H.X.; methodology, M.L., A.Z., and J.Z.; software, M.L.; validation, M.L., A.Z., and J.Z.; formal analysis, M.L.; investigation, M.L.; resources, M.L.; data curation, M.L.; writing—original draft preparation, M.L.; writing-review and editing, M.L.; visualization, M.L.; supervision, M.L., A.Z., J.Z., and H.X.; project administration, M.L.; funding acquisition, M.L. All authors have read and agreed to the published version of the manuscript.

Funding: This research was funded by National Natural Science Foundation of China, grant number 62105335. 
Conflicts of Interest: The authors declare no conflict of interest. The funders had no role in the design of the study; in the collection, analyses, or interpretation of data; in the writing of the manuscript; or in the decision to publish the results.

\section{References}

1. Lin-Hai, H. Coherent beam combination using a general model-based method. Chin. Phys. Lett. 2014, 31, $094205-094207$.

2. Wang, S.Q.; Wei, K.; Zheng, W.J.; Rao, C.H. First light on an adaptive optics system using a non-modulation pyramid wavefront sensor for a $1.8 \mathrm{~m}$ telescope. Chin. Opt. Lett. 2016, 14, 100101-100105. [CrossRef]

3. Xie, Z.L.; Ma, H.T.; Qi, B.; Ren, G.; Shi, J.; He, X.; Tan, Y.; Dong, L.; Wang, Z. Experimental demonstration of enhanced resolution of a Golay3 sparse-aperture telescope. Chin. Opt. Lett. 2017, 15, 041101-041104.

4. Bolcar, M.; Fienup, J. Sub-aperture piston phase diversity for segmented and multi-aperture systems. Appl. Opt. 2009, 48, A5-A12. [CrossRef]

5. Antonello, J.; Verhaegen, M. Modal-based phase retrieval for adaptive optics. J. Opt. Soc. Am. A 2015, 32, 1160-1170. [CrossRef]

6. Yeh, L.H.; Dong, J.; Zhong, J.; Tian, L.; Chen, M.; Tang, G.; Soltanolkotabi, M.; Waller, L. Experimental robustness of Fourier ptychography phase retrieval algorithms. Opt. Express 2015, 23, 33214-33240. [CrossRef] [PubMed]

7. Yang, Q.; Zhao, J.; Wang, M.; Jia, J. Wavefront sensorless adaptive optics based on the trust region method. Opt. Lett. 2015, 40, 1235-1237. [CrossRef]

8. Yang, P.; Ao, M.W.; Liu, Y.; Xu, B.; Jiang, W. Intracavity transverse modes controlled by a genetic algorithm based on zernike mode coefficients. Opt. Express 2007, 15, 17051-17062. [CrossRef]

9. Conkey, D.B.; Brown, A.N.; Caravaca-Aguirre, A.M.; Piestun, R. Genetic algorithm optimization for focusing through turbid media in noisy environments. Opt. Express 2012, 20, 4840-4849. [CrossRef]

10. Marsh, P.; Burns, D.; Girkin, J. Practical implementation of adaptive optics in multiphoton microscopy. Opt. Express 2003, 11, 1123-1130. [CrossRef] [PubMed]

11. Theofanidou, E.; Wilson, L.; Hossack, W.J.; Arlt, J. Spherical aberration correction for optical tweezers. Opt. Commun. 2004, 236, 145-150. [CrossRef]

12. Zommer, S.; Ribak, E.N.; Lipson, S.G.; Adler, J. Simulated annealing in ocular adaptive optics. Opt. Lett. 2006, 31, 939-941. [CrossRef]

13. Geng, C.; Luo, W.; Tan, Y.; Liu, H.; Mu, J.; Li, X.Y. Experimental demonstration of using divergence cost function in SPGD algorithm for coherent beam combining with tip/tilt control. Opt. Express 2013, 21, 25045-25055. [CrossRef]

14. Yang, H.Z.; Zhang, Z.; Wu, J. Performance comparison of wavefront-sensorless adaptive optics systems by using of the focal plane. Int. J. Opt. 2015, 2015, 985351. [CrossRef]

15. Ren, H.X.; Dong, B. Improved model-based wavefront sensorless adaptive optics for extended objects using N +2 images. Opt. Express 2020, 28, 14414-14427. [CrossRef]

16. Wen, L.H.; Yang, P.; Yang, K.J.; Chen, S.Q.; Wang, S.; Liu, W.J.; Xu, B. Synchronous model-based approach for wavefront sensorless adaptive optics system. Opt. Express 2017, 25, 20584-20597.

17. Yang, H.Z.; Soloviev, O.; Verhaegen, M. Model-based wavefront sensorless adaptive optics system for large aberrations and extended objects. Opt. Express 2015, 23, 24587-24601. [CrossRef] [PubMed]

18. Li, M.; Liu, X.; Zhang, A.; Xian, H. Telescopes alignment using the sharpness function method based on under-sampled images. IEEE PJ 2019, 1, 1-14.

19. Fu, Q.; Pott, J.; Shen, F.; Rao, C.H.; Li, X.Y. Stochastic parallel gradient descent optimization based on decoupling of the software and hardware. Opt. Commun. 2014, 310, 138-149. [CrossRef]

20. Dong, B.; Yu, J. Hybrid approach used for extended image-based wavefront sensor-less adaptive optics. Opt. Lett. 2015, 4, 041101-041110. [CrossRef]

21. Dong, B.; Ren, D.Q.; Zhang, X. Stochastic parallel gradient descent based adaptive optics used for a high contrast imaging coronagraph. Res. Astron. Astrophys. 2011, 11, 997-1002. [CrossRef]

22. Wang, X.L.; Zhou, P.; Ma, Y.X.; Leng, J.Y.; Xu, X.J.; Liu, Z.J. Active phasing a nine-element 1.14 kW all-fiber two-tone MOPA array using SPGD algorithm. Opt. Lett. 2011, 36, 3121-3123. [CrossRef]

23. Born, M.; Wolf, E. Principles of Optics, 2nd ed.; Cambridge University Press: Cambridge, UK, 1999.

24. Huang, L.H.; Rao, C.H. Wavefront sensorless adaptive optics: A general model-based approach. Opt. Express 2011, 19, 371-379.

25. Booth, M.J. Wavefront sensor-less adaptive optics: A model-based approach using sphere packings. Opt. Express 2006, 14, 1339-1352. [CrossRef] [PubMed]

26. Li, M.; Liu, X.; Zhang, A.; Xian, H. Telescope alignment based on the sharpness function of under-sampled images. Chin. Opt. Lett. 2019, 6, 061101-061105. [CrossRef] 